\title{
What is a mode in few mode fibers?: Proposal of MIMO- free mode division multiplexing using true eigenmodes
}

\author{
Yasuo Kokubun ${ }^{1 a)}$, Tatsuhiko Watanabe ${ }^{2}$, Seiya Miura², \\ and Ryo Kawata ${ }^{2}$ \\ ${ }^{1}$ Faculty of Engineering, Yokohama National University, \\ 79-5 Tokiwadai, Hodogaya-ku, Yokohama 240-8501, Japan \\ ${ }^{2}$ Graduate School of Engineering, Yokohama National University \\ a)kokubun-yasuo-sd@ynu.ac.jp
}

\begin{abstract}
The evolution of electromagnetic field profile of LP modes along with the propagation owing to the difference of propagation constants between constituent true eigenmodes is accurately analyzed. It is shown that the mode demultiplexer can't accurately discriminate the LP mode at the output end and so the MIMO-DSP is inevitable for the mode division multiplexed transmission using LP modes. From the accurate analysis, a transform matrix between LP modes and constituent true eigenmodes is derived and a novel method to configure true eigenmode multi/demultiplexer is proposed to realize MIMO-free transmission.
\end{abstract}

Keywords: few mode fiber, mode division multiplexing, true eigenmode, LP mode, mode multiplexer

Classification: Optical systems

\section{References}

[1] E. Snitzer: "Cylindrical dielectric waveguide modes," J. Opt. Soc. Am. 51 (1961) 491 (DOI: 10.1364/JOSA.51.000491).

[2] D. Gloge: "Weakly guiding fibers," Appl. Opt. 10 (1971) 2252 (DOI: 10.1364/ AO.10.002252).

[3] H. Kogelnik and P. J. Winzer: "Modal birefringence in weakly guiding fibers," J. Lightwave Technol. 30 (2012) 2240 (DOI: 10.1109/JLT.2012.2193872).

[4] J. Sakaguchi, et al.: "Large spatial channel (36-core $\times 3$ mode) heterogeneous few-mode multicore fiber," J. Lightwave Technol. 34 (2016) 93 (DOI: 10.1109/ JLT.2015.2481086).

[5] K. Shibahara, et al:: "Dense SDM (12-core $\times 3$-mode) transmission over $527 \mathrm{~km}$ with 33.2-ns mode-dispersion employing low-complexity parallel MIMO frequency-domain equalization," OFC2015 (2015) Th5C.3.

[6] D. Soma, et al:: "2.05 Peta-bit/s super-Nyquist-WDM SDM transmission using 9.8-km 6-mode 19-core fiber in full C band," ECOC2015 (2015) PDP.3.2 (DOI: 10.1109/ECOC.2015.7341686).

[7] J. von Hoyningen-Huene, et al:: "LCoS-based mode shaper for few-mode 
fiber," Opt. Express 21 (2013) 18097 (DOI: 10.1364/OE.21.018097).

[8] D. J. Richerdson: "Fiber amplifiers for SDM systems," OFC/NFOEC2013 (2013) OTu3G.1.

[9] E.-L. Lim, et al.: "Vector mode effects in few moded erbium doped fiber amplifiers," OFC/NFOEC2013 (2013) OTu3G.2.

[10] J. R. Carson, et al.: "Hyper-frequency wave guides - mathematical theory," Bell Syst. Tech. J. 15 (1936) 310 (DOI: 10.1002/j.1538-7305.1936.tb00734.x).

[11] Y. Kokubun: Lightwave Engineering (CRC Press, Optical Science and Engineering Series, 2012) 1st ed. 192 (ISBN-10:1420046489).

[12] Y. Kokubun, et al:: "Accurate analysis of crosstalk between $\mathrm{LP}_{11}$ degenerate modes due to offset connection using exact eigenmodes," to be presented at OECC/PS2016 (2016).

[13] J. Liu, et al.: "Demonstration of few mode fiber transmission link seeded by a silicon photonic integrated optical vortex emitter," ECOC2015 (2015) P.2.18 (DOI: 10.1109/ECOC.2015.7341730).

[14] N. Hanzawa, et al:: "Mode multi/demultiplexing with parallel waveguide for mode division multiplexed transmission," Opt. Express 22 (2014) 29321 (DOI: 10.1364/OE.22.029321).

\section{Introduction}

The true eigenmodes of round optical fiber are known as $\mathrm{HE}_{\ell m}$ and $\mathrm{EH}_{\ell m}\left(\mathrm{TE}_{0 m}\right.$ and $\mathrm{TM}_{0 m}$ when $\ell=0$ ) modes [1]. Since the propagation constants of $\mathrm{HE}_{\ell+1, m}$ and $\mathrm{EH}_{\ell-1, m}$ modes are quasi-degenerated, however, weakly guideing approximation and linearly polarized (LP) mode were proposed [2]. The linear combination of $\mathrm{HE}_{\ell+1, m}$ and $\mathrm{EH}_{\ell-1, m}$ quasi-degenerate modes can constitute a linearly polarized field profile, and this synthesized field profile is defined as $\mathrm{LP}_{\ell, m}$ mode. The polarization state of laser source used for the optical fiber transmission is linearly polarized, and so the excited electromagnetic field profile of optical fiber is also linearly polarized. Therefore, the LP mode is excited at the input end of few-mode fiber. Since the propagation constants of eigenmodes constituting an LP mode are not identical to each other [3], however, the electromagnetic field profile varies along with the propagation and is no longer the linear-polarized (LP) mode at the output end. In addition, the modal dispersion between true eigenmodes constituting an LP mode is as large as $1 \mathrm{~ns}$ for $100 \mathrm{~km}$ transmission [3]. Since the fundamental mode defined by $\mathrm{LP}_{01}$ mode is identical to $\mathrm{HE}_{11}$ mode, which is intrinsic linearly polarized mode, the single mode optical fiber transmission has been quit of the complexity of LP mode approximation.

On the other hand, after the recent rapid development of mode division multiplexing transmission, LP mode notation has been widely used in many ultra-large capacity long distance transmission experiments $[4,5,6]$. This is because the MIMO-DSP can reproduce the input channel by itterative numerical calculation, even though the output channels are mixed together. Therefore, the MIMO-DSP is inevitable for the mode division multiplexing using LP modes even when the transmission distance is short, e.g. less than $1 \mathrm{~km}$. The load of calculation of MIMO-DSP and the latency, however, will increase with the increase of the number of modes. 
To realize an MIMO-free mode division multiplexed transmission, an accurate analysis of behaviour of LP modes along with the propagation is needed, and there have been several reports that the intensity profile of $\mathrm{LP}_{11}^{\text {even }}$ mode evolves to become $\mathrm{LP}_{11}^{\text {odd }}$ mode along with the propagation and vice versa $[7,8,9]$. The period (beat length) of such evolution due to modal birefringence has been analyzed [3]. However, there has been no report on the accurate analysis of the evolution of electric field vector of LP modes.

In this study, we analyze accurately the evolution of electromagnetic field of LP modes along with the propagation and show that the field can be expressed by the localized elliptical polarization. Next the transform matrix between LP modes and true eigenmodes is derived and a novel method to constitute "true eigenmode multi/demultiplexer" by the combination of in-phase/reversed-phase $2 \times 2$ circuit and the conventional LP-mode multi/demultiplexer.

\section{Transform matrix between LP modes and true eigenmodes}

The eigenmode of round dielectric waveguide was first analyzed by Carton et al. [10], and the definition of HE, EH, TE, and TM modes was given by Snitzer [1]. In the following analysis, we use the definition and notation of true eigenmodes by Snitzer. Although Snitzer used the time and $z$ dependent term as $\exp [i(\beta z-\omega t)]$, however, we use the notation $\exp [j(\omega t-\beta z)]$.

\subsection{Electromagnetic field of LP mode consisting of true eigenmodes}

Let us assume a step-index round optical fiber of which index profile is given by

$$
n^{2}(r)= \begin{cases}n_{1}^{2} & (r \leq a) \\ n_{2}^{2}=n_{1}^{2}[1-2 \Delta] & (r>a)\end{cases}
$$

where $n_{1}$ and $n_{2}$ are the refractive indexes of core and cladding, respectively, $a$ is the core radius, and $\Delta$ is the relative index difference. The solution $E_{z}$ and $H_{z}$ of vector wave equation in the cylindrical cordinate system is expressed in terms of Bessel function inside the core as follows.

$$
\begin{aligned}
& E_{z}=A_{\ell} J_{\ell}(\kappa r) \cos \left(\ell \theta+\varphi_{\ell}\right) \exp [j(\omega t-\beta z)] \\
& H_{z}=B_{\ell} J_{\ell}(\kappa r) \cos \left(\ell \theta+\psi_{\ell}\right) \exp [j(\omega t-\beta z)]
\end{aligned}
$$

where $A_{\ell}$ and $B_{\ell}$ are the amplitudes, $\ell$ is the mode number in the azimuth direction, and $\varphi_{\ell}$ and $\psi_{\ell}$ are azimuth phases, which represent even and odd modes for degenerate modes. Here $\kappa$ is given by $\kappa=\sqrt{k_{0}^{2} n_{1}^{2}-\beta^{2}}$, and $\varphi_{\ell}-\psi_{\ell}= \pm \frac{\pi}{2}$ [1]. Since $E_{z}$ and $H_{z}$ in the cladding are obtained by replacing $J_{\ell}(\kappa r)$ by $\frac{J_{\ell}(\kappa a)}{K_{\ell}(\gamma a)} K_{\ell}(\gamma r)$, where $\gamma=\sqrt{\beta^{2}-k_{0}^{2} n_{2}^{2}}$ and $K_{\ell}(x)$ is the modified Bessel function of second kind, we discuss only the field profile inside the core.

The transverse components $E_{r}, E_{\theta}, H_{r}$ and $H_{\theta}$ are derived from the longitudinal components $E_{z}$ and $H_{z}$. Since only the electric components $E_{r}$ and $E_{\theta}$, are required to describe the polarization state of modes, we derive $E_{r}$ and $E_{\theta}$ from Eq. (2) as follows. 


$$
\begin{aligned}
E_{r}^{(\ell)} & =-j \frac{\beta}{\kappa}\left[\frac{1-P}{2} J_{\ell-1}(\kappa r)-\frac{1+P}{2} J_{\ell+1}(\kappa r)\right] F_{c} \\
E_{\theta}{ }^{(\ell)} & =-j \frac{\beta}{\kappa}\left[-\frac{1-P}{2} J_{\ell-1}(\kappa r)-\frac{1+P}{2} J_{\ell+1}(\kappa r)\right] F_{S}
\end{aligned}
$$

where $F_{c}$ and $F_{s}$ are defined by

$$
\begin{aligned}
& F_{c}=A_{\ell} \cos \left(\ell \theta+\varphi_{\ell}\right) \exp [j(\omega t-\beta z)] \\
& F_{s}=A_{\ell} \sin \left(\ell \theta+\varphi_{\ell}\right) \exp [j(\omega t-\beta z)]
\end{aligned}
$$

The parameter $P$ was introduced by Snitzer [1] and is defined by

$$
P=-\frac{\omega \mu_{0}}{\beta} \frac{H_{z}}{E_{z}}=-\frac{\omega \mu_{0}}{\beta} \frac{B_{\ell} \cos \left(\ell \theta+\psi_{\ell}\right)}{A_{\ell} \sin \left(\ell \theta+\varphi_{\ell}\right)}
$$

The true eigenmodes are classified into HE and EH modes in terms of the parameter $P$ as shown in Table I.

Table I. Classification of true eigenmodes in terms of $P[1]$

\begin{tabular}{|c|c|c|}
\hline Mode label & At cutoff & Far from cutoff \\
\hline $\mathrm{HE}_{\ell m}(\ell>1)$ & $P=-1$ & $P=-1$ \\
\hline $\mathrm{EH}_{\ell m}(\ell>1)$ & $P=\frac{n_{1}^{2}}{n_{2}^{2}} \simeq 1$ & $P=1$ \\
$\mathrm{TM}_{0 m}$ & $P=0$ & $P=0$ \\
$\mathrm{TE}_{0 m}$ & $P=\infty$ & $P=\infty$ \\
\hline
\end{tabular}

On the other hand, $\mathrm{LP}_{\mu m}$ mode consists of $\mathrm{HE}_{\mu+1 m}$ and $\mathrm{EH}_{\mu-1 m}$ modes $\left(\mathrm{TE}_{0 m}\right.$ and $\mathrm{TM}_{0 m}$ modes when $\mu=1$ ), and the electric fields of HE and EH modes are superimposed to form a linearly polarized electric field by regulating the azimuth phases $\varphi_{\ell}$ and $\psi_{\ell}$ [2]. The $x$ and $y$ components of electric field of true eigenmode are derived from Eqs. (4) and (5) to be

$$
\begin{aligned}
E_{x}^{(\ell)}=-j & \frac{\beta}{\kappa} A_{\ell} e^{j\left(\omega t-\beta_{\ell} z\right)} \\
\times & {\left[\frac{1-P}{2} J_{\ell-1}(\kappa r) \cos \left\{(\ell-1) \theta+\varphi_{\ell}\right\}\right.} \\
& \left.-\frac{1+P}{2} J_{\ell+1}(\kappa r) \cos \left\{(\ell+1) \theta+\varphi_{\ell}\right\}\right] \\
E_{y}{ }^{(\ell)}=- & j \frac{\beta}{\kappa} A_{\ell} e^{j\left(\omega t-\beta_{\ell} z\right)} \\
\times & {\left[-\frac{1-P}{2} J_{\ell-1}(\kappa r) \sin \left\{(\ell-1) \theta+\varphi_{\ell}\right\}\right.} \\
& \left.-\frac{1+P}{2} J_{\ell+1}(\kappa r) \sin \left\{(\ell+1) \theta+\varphi_{\ell}\right\}\right]
\end{aligned}
$$

using the cordinate transform matrix. Then by substituting the value of $P$ given in Table I into Eqs. (9) and (10), the $x$ and $y$ components of electric field of HE, EH, TE, and TM modes are derived as follows. 
a. $\mathrm{HE}_{\mu+1, m}$ mode (component of $\mathrm{LP}_{\mu, m}$ mode, $\left.P=-1\right)$

$$
\begin{aligned}
& E_{x}{ }^{(\mu+1)}=-j \frac{\beta}{\kappa} A_{\mu+1} e^{j\left(\omega t-\beta_{\mu+1} z\right)} J_{\mu}(\kappa r) \cos \left(\mu \theta+\varphi_{\mu+1}\right) \\
& E_{y}{ }^{(\mu+1)}=j \frac{\beta}{\kappa} A_{\mu+1} e^{j\left(\omega t-\beta_{\mu+1} z\right)} J_{\mu}(\kappa r) \sin \left(\mu \theta+\varphi_{\mu+1}\right)
\end{aligned}
$$

b. $\mathrm{EH}_{\mu-1, m}$ mode (component of $\mathrm{LP}_{\mu, m}$ mode, $P=+1$ )

$$
\begin{aligned}
& E_{x}^{(\mu-1)}=j \frac{\beta}{\kappa} A_{\mu-1} e^{j\left(\omega t-\beta_{\mu-1} z\right)} J_{\mu}(\kappa r) \cos \left(\mu \theta+\varphi_{\mu-1}\right) \\
& E_{y}{ }^{(\mu-1)}=j \frac{\beta}{\kappa} A_{\mu-1} e^{j\left(\omega t-\beta_{\mu-1} z\right)} J_{\mu}(\kappa r) \sin \left(\mu \theta+\varphi_{\mu-1}\right)
\end{aligned}
$$

c. $\mathrm{TM}_{0, m}$ mode (component of $\mathrm{LP}_{1, m}$ mode, $P=0$ )

d. $\mathrm{TE}_{0, m}$ mode (component of $\mathrm{LP}_{1, m}$ mode, $P=\infty$ )

In the case of TE and TM modes, since $P \neq \pm 1$, Eqs. (9) and (10) can not be used and we need to derive the expressions before reaching Eqs. (4) and (5) in Ref. [1]. Then the derived equations are

$$
\begin{aligned}
& E_{x}{ }^{(0)}=j \frac{\beta}{\kappa} A_{0} e^{j\left(\omega t-\beta_{0} z\right)} J_{1}(\kappa r) \cos \left(\theta+\varphi_{0}\right) \\
& E_{y}{ }^{(0)}=j \frac{\beta}{\kappa} A_{0} e^{j\left(\omega t-\beta_{0} z\right)} J_{1}(\kappa r) \sin \left(\theta+\varphi_{0}\right)
\end{aligned}
$$

where $\varphi_{0}=0$ and $\varphi_{0}=\frac{\pi}{2}$ correspond to $\mathrm{TM}_{0, m}$ and $\mathrm{TE}_{0, m}$ modes, respectively, and it should be noted that the propagation constant $\beta_{0}$ differs for TM and TE modes, i.e. $\mathrm{TE}_{0, m}$ and $\mathrm{TM}_{0, m}$ modes are not degenerated [3].

\subsection{Synthesis of electric field of LP mode in terms of true eigenmodes}

Let us suppose the two mode region of step-index fiber corresponding to $V \leq 3.81$, supporting $\mathrm{LP}_{01}$ and $\mathrm{LP}_{11}$ modes. Since there are two orthogonal polarizations ( $x$ and $y$ polarizations) and two orthogonal field profiles, i.e. even and odd modes for $\mathrm{LP}_{11}$ modes, six orthogonal modes are actually supported as summarized in Table II. The fundamental $\mathrm{LP}_{01}$ mode corresponds to $\mathrm{HE}_{11}$ mode, and the first order $\mathrm{LP}_{11}$ mode consists of $\mathrm{HE}_{21}^{\text {even }}, \mathrm{HE}_{21}^{\text {odd }}, \mathrm{TM}_{01}$, and $\mathrm{TE}_{01}$ modes.

Table II. Definition of degenerated $\mathrm{LP}_{01}, \mathrm{LP}_{11}^{\text {even }}$, and $\mathrm{LP}_{11}^{\text {odd }}$ modes

\begin{tabular}{|l|l|l|l|}
\hline$x_{k}{ }^{y} z$ & $\mathrm{LP}_{01}$ & $\mathrm{LP}_{11}^{\text {even }}$ & $\mathrm{LP}_{11}^{\text {odd }}$ \\
\hline $\begin{array}{l}x- \\
\text { polarization } \\
\text { polarization }\end{array}$ & $\longrightarrow$ & $\longrightarrow$ \\
\hline
\end{tabular}




\subsection{1 $x$ polarized $\mathrm{LP}_{11}^{\text {even }}$ mode input (TMH group)}

The sum of Eq. (11) for $\mathrm{HE}_{21}$ mode with $\varphi_{2}=0$ (corresponding to $\mathrm{HE}_{21}^{\text {even }}$ mode) and Eq. (15) for $\mathrm{TM}_{01}$ mode with $\varphi_{0}=0$ gives the $x$ component of electric field of $\mathrm{LP}_{11}^{\text {even }}$ mode at the input end. In the same way, the sum of Eq. (12) and Eq. (16) gives the $y$ component of electric field of $\mathrm{LP}_{11}^{\text {even }}$ mode at the input end. These relations can be seen from the vectorial summation of the electric field profiles as illustrated in Figs. 6.7(a) and (b) in Reference [11]. When the polarization of input beam is $x$ polarization, $-A_{2}=A_{0}=A_{L P 11}^{\mathrm{e}}$ should be satisfied to make $y$ component zero. In this case, the $x$ and $y$ components of electric field at the propagation distance $z$ is expressed by

$$
\begin{aligned}
& E_{x}^{\mathrm{LP} 11-\mathrm{e}}=j \frac{\beta_{a M}}{\kappa} A_{L P 11}^{\mathrm{e}} e^{j\left(\omega t-\beta_{a M} \cdot z\right)} J_{1}(\kappa r) \cos (\theta)\left[2 \cos \left(\delta \beta_{M} \cdot z\right)\right] \\
& E_{y}^{\mathrm{LP} 11-\mathrm{e}}=j \frac{\beta_{a M}}{\kappa} A_{L P 11}^{\mathrm{e}} e^{j\left(\omega t-\beta_{a M} \cdot z\right)} J_{1}(\kappa r) \sin (\theta)\left[2 j \sin \left(\delta \beta_{M} \cdot z\right)\right]
\end{aligned}
$$

Here $\beta_{a M}$ is the average of propagation constants of $\mathrm{HE}_{21}$ and $\mathrm{TM}_{01}$ modes and $\delta \beta_{M}$ is the half of the difference of these propagation constants, which are defined by

$$
\begin{aligned}
\beta_{a M} & =\frac{\beta_{H E 21}+\beta_{T M 01}}{2} \\
\delta \beta_{M} & =\frac{\beta_{H E 21}-\beta_{T M 01}}{2}
\end{aligned}
$$

The intensity profile ( $z$ component of complex Poynting vector) is derived from Eqs. (17) and (18) and is expressed by

$$
\begin{aligned}
\tilde{S}_{z}= & \frac{2 \beta_{a M}^{3}}{\omega \mu_{0} \kappa^{2}}\left(A_{L P 11}^{\mathrm{e}}\right)^{2} J_{1}^{2}(\kappa r) \\
& \times\left[\cos ^{2}\left(\delta \beta_{M} \cdot z\right) \cos ^{2}(\theta)+\sin ^{2}\left(\delta \beta_{M} \cdot z\right) \sin ^{2}(\theta)\right]
\end{aligned}
$$

It can be seen from Eqs. (17) and (18) that the electric field profile is expressed by a locally elliptical polarization of which ellipticity and the direction of rotation of polarization depend on position in the transverse cross section, because the phase of $y$ component in Eq. (18) differs from that of $x$ component in Eq. (17) by $\pm \frac{\pi}{2}$. This fact has not been shown in References [3] and [7, 8, 9].

On the other hand, the intensity profile of $\mathrm{LP}_{11-x}^{\text {even }}$ mode evolves into that of $\mathrm{LP}_{11-y}^{\text {odd }}$ mode via a torus-shape intensity profile, and this change is periodic with respect to the propagation distance. Therefore, $\mathrm{LP}_{11-x}^{\text {even }}$ mode and $\mathrm{LP}_{11-y}^{\text {odd }}$ mode belong to the same mode group called TMH mode group [3], of which constituent true eigenmodes are $\mathrm{HE}_{21}^{\text {even }}$ and $\mathrm{TM}_{01}$ modes. Fig. 1 shows the evolution of intensity profile of $\mathrm{LP}_{11-x}^{\text {even }}$ mode to $\mathrm{LP}_{11-y}^{\text {odd }}$ mode, and Fig. 2 illustrates the detailed polarization state distribution together with the intensity profile at the propagation distance satisfying $\delta \beta_{M} \cdot z=\frac{\pi}{4}+2 \pi N(N=$ integer $)$.

The following conclusions are derived from these facts.

(a) The electromagnetic field profile at the output end of FMF is not any longer that of the LP mode even if the linearly polarized light is incident on the input end of FMF.

(b) When $\mathrm{LP}_{11-x}^{\text {even }}$ mode is excited at the input end of FMF, the field profile evolves into $\mathrm{LP}_{11-y}^{\text {odd }}$ mode at the propagation distance given by 


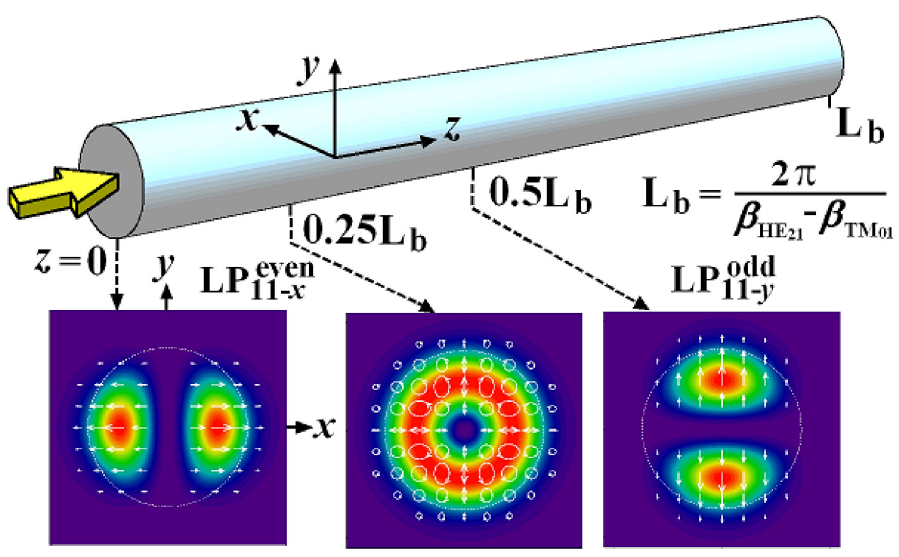

Fig. 1. Evolution of field profile of $\mathrm{LP}_{11-x}^{\text {even }}$ mode to $\mathrm{LP}_{11-y}^{\text {odd }}$ mode

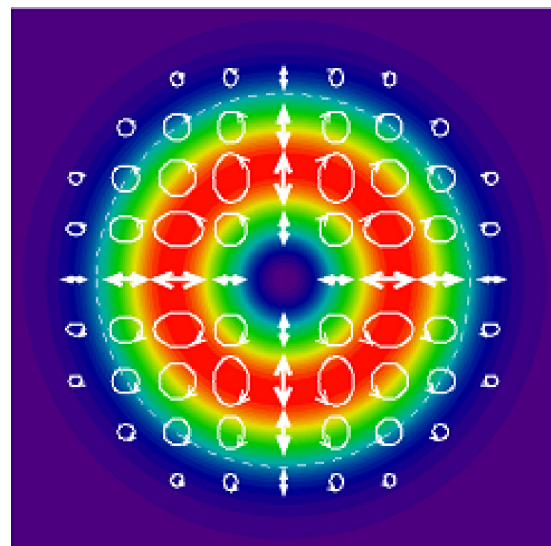

Fig. 2. Polarization state distribution of $\mathrm{TMH}$ mode group at the middle point of evolution from $\mathrm{LP}_{11-x}^{\text {even }}$ mode to $\mathrm{LP}_{11-y}^{\text {odd }}$ mode

$$
\frac{L_{b}^{\mathrm{TMH}}}{2}=\frac{\pi}{\delta \beta_{M}},
$$

and this change is repeated with the period of $L_{b}^{\mathrm{TMH}}$, even if the fiber is ideally straight and circular.

(c) Therefore, the analysis and measurement of modal characteristics of FMFs without any information on the polarization state don't make any sense. The crosstalk analysis due to the offset splicing also should take into account this modal evolution [12].

\subsection{2 $x$ polarized LP ${ }_{11}^{\text {odd }}$ mode input (TEH group)}

In the same way as subsection 2.2.1, the sum of Eq. (11) for $\mathrm{HE}_{21}$ mode with $\varphi_{2}=\frac{\pi}{2}$ (corresponding to $\mathrm{HE}_{21}^{\text {odd }}$ mode) and Eq. (15) for $\mathrm{TE}_{01}$ mode with $\varphi_{0}=\frac{\pi}{2}$ gives the $x$ component of electric field of $\mathrm{LP}_{11}^{\text {odd }}$ mode at the input end. On the other hand, the sum of Eq. (12) and Eq. (16) gives the $y$ component of electric field of $\mathrm{LP}_{11}^{\text {odd }}$ mode at the input end. When the polarization of input beam is $x$ polarization, $A_{2}=-A_{0}=A_{L P 11}^{\mathrm{o}}$ should be satisfied to make $y$ component zero. In this case, the $x$ and $y$ components of electric field at the propagation distance $z$ is expressed by 


$$
\begin{aligned}
& E_{x}^{\mathrm{LP} 11-\mathrm{o}}=j \frac{\beta_{a E}}{\kappa} A_{L P 11}^{\mathrm{o}} e^{j\left(\omega t-\beta_{a E} \cdot z\right)} J_{1}(\kappa r) \sin (\theta)\left[2 \cos \left(\delta \beta_{E} \cdot z\right)\right] \\
& E_{y}^{\mathrm{LP} 11-\mathrm{o}}=j \frac{\beta_{a E}}{\kappa} A_{L P 11}^{\mathrm{o}} e^{j\left(\omega t-\beta_{a E} \cdot z\right)} J_{1}(\kappa r) \cos (\theta)\left[-2 j \sin \left(\delta \beta_{E} \cdot z\right)\right]
\end{aligned}
$$

where $\beta_{a E}$ and $\delta \beta_{E}$ are defined by

$$
\begin{gathered}
\beta_{a E}=\frac{\beta_{H E 21}+\beta_{T E 01}}{2} \\
\delta \beta_{E}=\frac{\beta_{H E 21}-\beta_{T E 01}}{2}
\end{gathered}
$$

The evolution of intensity profile is shown in Fig. 3, and the detailed polarization state distribution together with the intensity profile at the propagation distance satisfying $\delta \beta_{E} \cdot z=\frac{\pi}{4}+2 \pi N(N=$ integer $)$ is illustrated in Fig. 4.

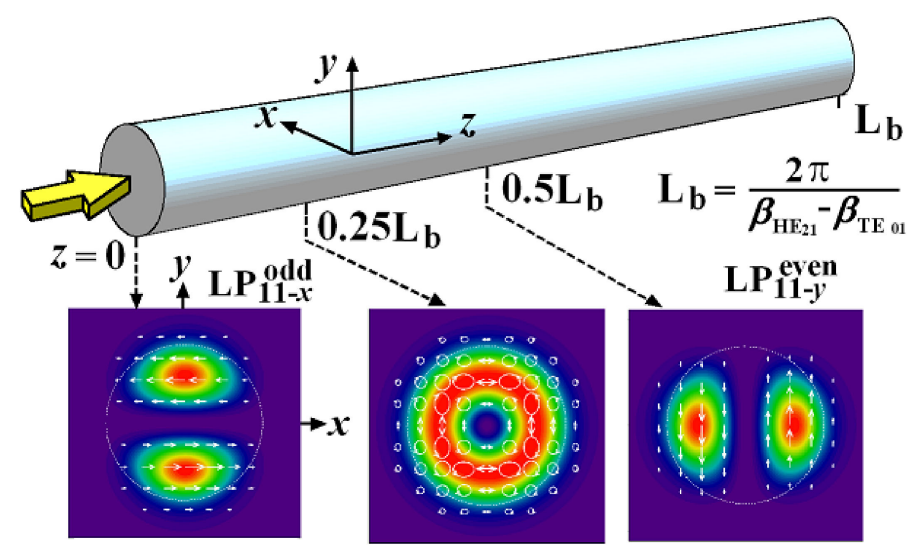

Fig. 3. Evolution of field profile of $\mathrm{LP}_{11-x}^{\text {odd }}$ to $\mathrm{LP}_{11-y}^{\text {even }}$ mode

The transform matrix between $\mathrm{LP}_{11}$ modes and true eigenmodes is derived from Eqs. (11), (12), (15), (16), (17), (18), (23), and (24) as follows.

$$
\left[\begin{array}{c}
\mathrm{LP}_{11-x}^{\text {even }} \\
\mathrm{LP}_{11-y}^{\text {odd }} \\
\mathrm{LP}_{11-x}^{\text {odd }} \\
\mathrm{LP}_{11-y}^{\text {even }}
\end{array}\right]=\frac{1}{\sqrt{2}}\left[\begin{array}{cccc}
1 & -1 & 0 & 0 \\
1 & 1 & 0 & 0 \\
0 & 0 & 1 & -1 \\
0 & 0 & 1 & 1
\end{array}\right] \cdot\left[\begin{array}{c}
\mathrm{TM}_{01} \\
\mathrm{HE}_{21}^{\text {even }} \\
\mathrm{HE}_{21}^{\text {odd }} \\
\mathrm{TE}_{01}
\end{array}\right]
$$

\subsubsection{Period of modal evolution}

The period of modal evolution between $\mathrm{LP}_{11-x}^{\text {even }}$ and $\mathrm{LP}_{11-y}^{\text {odd }}$ is greater by one order than that between $\mathrm{LP}_{11-x}^{\text {odd }}$ and $\mathrm{LP}_{11-y}^{\text {even }}$, because the values of $\delta \beta_{M}$ and $\delta \beta_{E}$ are very different $[3,8]$. The period of modal evolution (beat length) is given by [3]

$$
L_{b}{ }^{(v)}=\frac{n_{\mathrm{eq}} \lambda}{n_{1}^{2} \Delta^{2} B_{v}(V)}, \quad(v=\mathrm{TMH} \text { or TEH })
$$

where $B_{\mathrm{TMH}}(V)$ and $B_{\mathrm{TEH}}(V)$ are normalized differences of propagation constants which are related to $\delta \beta_{M}$ and $\delta \beta_{E}$ by 


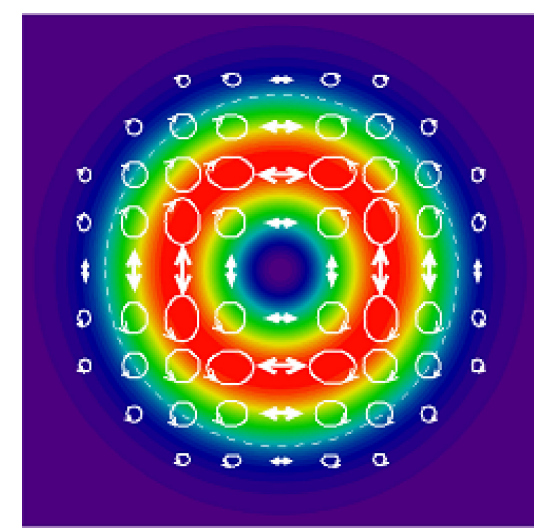

Fig. 4. Polarization state distribution of TEH mode group at the middle point of evolution from $\mathrm{LP}_{11-x}^{\text {odd }}$ mode to $\mathrm{LP}_{11-y}^{\text {even }}$ mode

$$
B_{\mathrm{TMH}}(V)=\frac{2 n_{\mathrm{eq}}}{n_{1}^{2} \Delta^{2}} \delta \beta_{M}, \quad B_{\mathrm{TEH}}(V)=\frac{2 n_{\mathrm{eq}}}{n_{1}^{2} \Delta^{2}} \delta \beta_{E} .
$$

Since the formulas for calculating $\delta \beta_{M}$ and $\delta \beta_{E}$ have been derived in Ref. [3] in detail, only the calculated results are shown here. The normalized difference of propagation constant and the period of mode evolution are plotted as a function of $V$ parameter as shown in Fig. 5 and Fig. 6, respectively. It can be seen from Fig. 6 that the period of mode evolution of TMH mode group depends strongly on $V$ and varies from several tens centimeters to several meters, while that of TEH mode group is in the order of several tens centimeters. Therefore, the behavior of field profile is also quite different for TMH and TEH mode groups, because the wavelength (frequency) dependence of modal evolution is quite different.

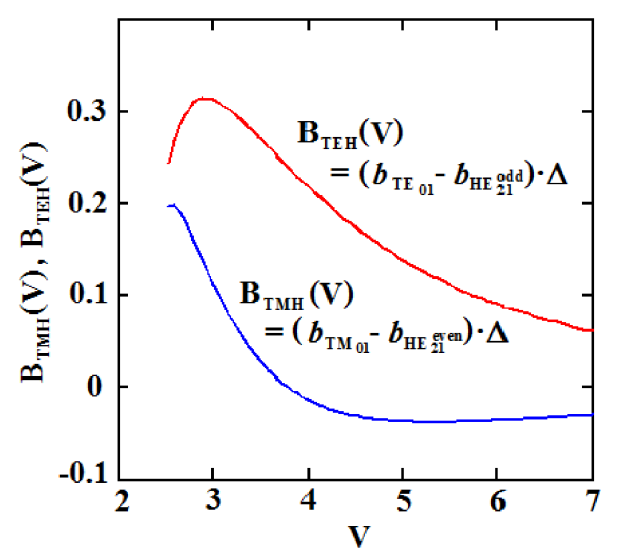

Fig. 5. $\quad V$ dependence of normalized difference of propagation constant 


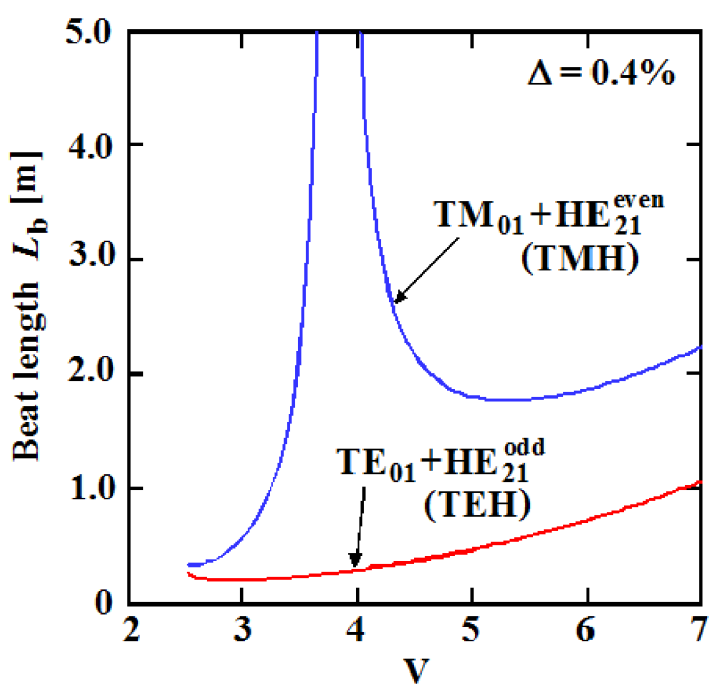

Fig. 6. $\quad V$ dependence of period of mode evolution $(\Delta=0.4 \%)$

\section{Proposal of mode multiplexed transmission using true eigen- modes}

Since the transmission channels using LP modes are mixed together as described above, the MIMO-DSP is inevitable for the mode division multiplexing using LP modes even when the transmission distance is short, e.g. less than $10 \mathrm{~m}$. In addition, the modal dispersion between true eigen-modes constituting an LP mode is as large as $1 \mathrm{~ns}$ for $100 \mathrm{~km}$ transmission [3]. To overcome these problems and to realize a MIMO-free transmission, a novel mode multiplexed transmission using true eigenmodes is needed. It has been difficult, however, to excite selectively true eigenmodes except the fundamental mode because higher order modes like $\mathrm{TE}_{01}$, $\mathrm{TM}_{01}, \mathrm{HE}_{21}^{\text {even }}$, and $\mathrm{HE}_{21}^{\text {odd }}$ modes have complex field profiles such as azimuth, radial, and bent looped electric field [1]. Although a TE and TM modes exciter has been proposed [13], this device can not excite $\mathrm{HE}_{21}$ modes and has a problem of strong wavelength dependence because of the microring resonator structure with corrugated side-wall.

Instead of exciting directly higher order true eigenmodes, we can utilize the relation between LP modes and true eigenmodes expressed by the transform matrix Eq. (27). The inverse matrix of Eq. (27) is given by

$$
\left[\begin{array}{c}
\mathrm{TM}_{01} \\
\mathrm{HE}_{21}^{\text {even }} \\
\mathrm{HE}_{21}^{\text {odd }} \\
\mathrm{TE}_{01}
\end{array}\right]=\frac{1}{\sqrt{2}}\left[\begin{array}{cccc}
1 & 1 & 0 & 0 \\
-1 & 1 & 0 & 0 \\
0 & 0 & 1 & 1 \\
0 & 0 & -1 & 1
\end{array}\right] \cdot\left[\begin{array}{c}
\mathrm{LP}_{11-x}^{\text {even }} \\
\mathrm{LP}_{11-y}^{\text {odd }} \\
\mathrm{LP}_{11-x}^{\text {odd }} \\
\mathrm{LP}_{11-y}^{\text {even }}
\end{array}\right]
$$

It can be seen from the first and second lines of Eq. (30) that $\mathrm{TM}_{01}$ mode can be synthesized by adding $\mathrm{LP}_{11-x}^{\text {even }}$ and $\mathrm{LP}_{11-y}^{\text {odd }}$ modes in-phase, and $\mathrm{HE}_{21}^{\text {even }}$ mode can be synthesized by adding $\mathrm{LP}_{11-x}^{\text {even }}$ and $\mathrm{LP}_{11-y}^{\text {odd }}$ with reversed phase. As for the TEH mode group, the similar synthesis is possible by adding $\mathrm{LP}_{11-x}^{\text {odd }}$ and $\mathrm{LP}_{11-y}^{\text {even }}$ modes in-phase and reversed phase to excite $\mathrm{HE}_{21}^{\text {odd }}$ mode and $\mathrm{TE}_{01}$ mode, respectively. 
Utilizing the above relationship, a novel true eigenmode multi/demultiplexer can be configured by the combination of in-phase/reversed-phase $2 \times 2$ circuit and the conventional LP-mode multi/demultiplexer as shown in Fig. 7. Asymmetric $\mathrm{X}$-shaped mixing and branching circuit and/or asymmetric directional coupler can be used as the in-phase/reversed-phase $2 \times 2$ circuit as shown in Fig. 7. As the LP-mode multi/demultiplexer, a PLC-type mode multi/demultiplexer with mode rotator [14] can be used. The mode rotator can act as the polarization rotator as well as the mode rotator. True eigenmode multi/demultiplexer for higher order modes will be possible because the transform matrix between higher order LP modes and constituent true eigenmodes is similar to that expressed in Eq. (30).

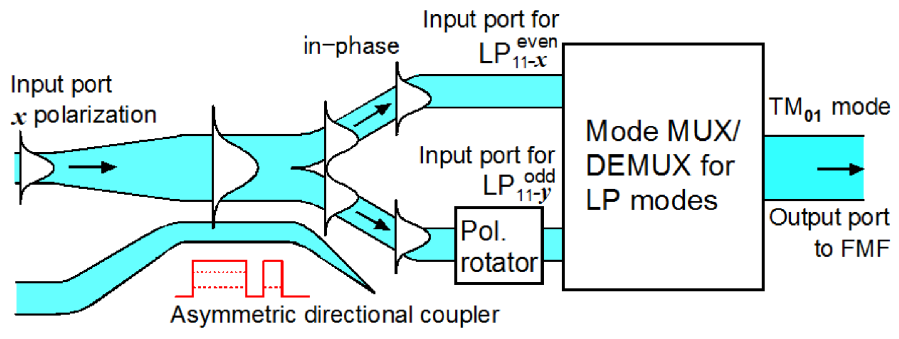

(a) $\mathrm{TM}_{01}$ mode multiplexing

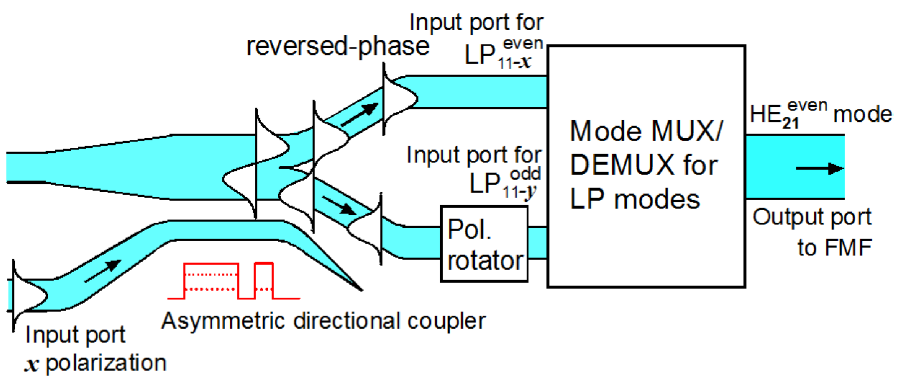

(b) $\mathrm{HE}_{21}^{\text {even }}$ mode multiplexing

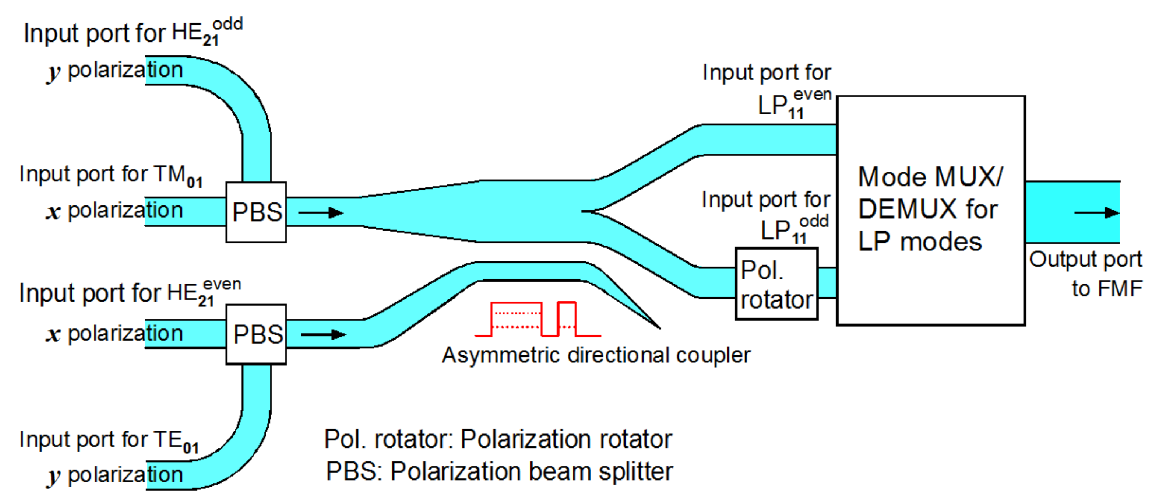

(c) All four eigenmodes multiplexing

Fig. 7. True eigenmode multi/demultiplexer

\section{Conclusion}


MIMO-free transmission as long as the mode mixing is negligible. The true eigenmode multi/demultiplexer will enable such MIMO-free MDM transmission.

\section{Acknowledgments}

This work was supported by the National Institute of Information and Communications Technology (NICT), Japan under "R\&D of Innovative Optical Fiber and Communication Technology". 